\title{
Analysis of 3D Plastic Deformation in Vertical Rolling Based on Global Weighted Velocity Field
}

\section{Boxin Yang}

East China University of Science and Technology

\section{Haojie Xu}

East China University of Science and Technology

Qi An ( $D$ anqi@ecust.edu.cn )

East China University of Science and Technology

\section{Research Article}

Keywords: Vertical Rolling, Energy Method, Weighted Velocity Field, 3D Plastic Deformation

Posted Date: January 13th, 2022

DOI: https://doi.org/10.21203/rs.3.rs-1234080/v1

License: (c) (1) This work is licensed under a Creative Commons Attribution 4.0 International License. Read Full License

Version of Record: A version of this preprint was published at The International Journal of Advanced Manufacturing Technology on April 14th, 2022. See the published version at https://doi.org/10.1007/s00170-022-09190-4. 


\title{
Analysis of 3D Plastic Deformation in Vertical Rolling Based on Global Weighted Velocity
}

\section{Field}

\author{
Boxin Yang ${ }^{1}$, Haojie $\mathrm{Xu}^{1}$, Qi An ${ }^{1, *}$ \\ 1 School of Mechanical and Power Engineering, East China University of Science and Technology, \\ Shanghai, 200237, PR China \\ Corresponding author: Qi An (anqi@ecust.edu.cn)
}

\begin{abstract}
Energy method is an essential theoretical approach to analyze plastic forming, which is widely used in rolling. An analysis model for vertical rolling process is established according to energy theory. By using global weighted method firstly, the $3 \mathrm{D}$ continuous velocity field, strain rate field and the corresponding power functional are proposed. The unknown variables are solved numerically based on the principle of minimum energy. Then, deformation parameters and rolling force are determined. The analysis on specific examples with the width reduction rate of $0.03 \sim 0.05$ shows that the theoretical prediction value of weighted model is in good agreement with experimental results. Moreover, the effects of several shape and rolling parameters on rolling force, rolling power and edge deformation are studied. Both the width reduction rate and initial slab thickness have significant influences on dog-bone size and rolling force. A wider slab slightly increases the nonuniformity of dog-bone deformation. An increase of vertical roller radius can weaken the edge deformation.
\end{abstract}

Keywords: Vertical Rolling, Energy Method, Weighted Velocity Field, 3D Plastic Deformation

\section{Introduction}

During continuous hot rolling process, the vertical rolling is often arranged at initial stage to fix the width and improve the rectangular degree of the casting slab. Quite distinct from the entire and uniform plastic deformation in flat rolling, the nonuniform plastic deformation only occurs at the edge part, which leads to the complex rules of rolling force and plastic deformation. In order to guarantee the quality requirement of steel, establishing an accurate mathematical model for vertical rolling is of great significance.

Variational principle, which can be summarized as presenting the kinematically admissible velocity field at first, and then determining the real plastic flow by minimizing the power functional, is widely used in rolling research. Kobayashi et. al [1] established the kinematically admissible velocity field based on energy method, and obtained rolling force and width spread of 3D rolling deformation numerically. Kato et. al [2] weighted the velocity components in $\mathrm{x}$ and $\mathrm{z}$ directions, and obtained the local weighted velocity field with the corresponding numerical results of rolling force and torque. By using upper bound approach, Avitzur et. al established continuous deformation [3-4] and triangular [5] velocity fields in strip rolling respectively. The total power functional contains four independent process parameters (relative thickness, reduction, friction and net front-back tension). Dong et. al [6] built a simplified 3D theoretical model for rail rolling by universal mill firstly. The kinematically admissible velocity field of the web, head, and base of rail was determined respectively. The experiment results verified the reliable and feasible of the model. Zhang $\mathrm{J} 1$ et. al [7] proposed a mixed analytical-numerical method to predict the velocity field and strain distribution during multi-pass plate hot rolling. After solving the velocity field, strain rate field and rolling force, the thermal model coupling with plastic deformation is exploited through series function solution to determine temperature distribution and calculate the flow stress. Based on elastic and plastic mechanics, Sun et. al [8] gave a 
hyperbolic sine velocity field to predict rolling force in tandem cold rolling. The theoretical prediction was compared with other researchers' models and on-line measured result. For the analysis of hot strip rolling, Zhang D H et. al [9] simplified the weighted velocity field and presented the analytical expression of total power functional. The effects of various rolling conditions such as thickness reduction, friction factor, shape factor, upon separating force, location of neutral angle and stress state coefficient were discussed systematically. Cao et. al [10] set up a logarithmic velocity field and the corresponding total power was obtained by using linear equal area (EA) yield criterion and co-line vector inner product method. The rolling force and torque are received according to the principle of minimum energy. Zhang S H et. al [11] proposed a 3D velocity field for plate rolling by global weighted method. After deduced the relationship between weighting coefficient and width spread, the rolling force and energy parameters are calculated. Finally, a series of laws between mechanical parameters and shape parameters are put forward. In order to obtain the analytical equation of power functional in hot strip finishing mill, Ma et. al [12] assumed a sine velocity field and determined average deformation resistance by thermo-mechanical coupled analysis. The effects of various rolling conditions, such as thickness reduction, friction factor and shape factor, on roll force, location of neutral angle and stress-state coefficient are discussed systematically. Peng et. al [13-14] proposed parabolic and cylindrical velocity fields for hot tandem and cold rolling respectively. The analysis solution of mechanics parameters is obtained and discussed by minimizing the total power. Based on a tangent velocity and the linear mean yield (MY) criterion, Li S et. al [15] predicted the rolling force in hot strip rolling process and compared the result with the experimental data.

Recently, energy method has been applied in the theoretical research on vertical rolling process. Yun et. al [16] proposed a dog-bone model with exponential function and quartic function, and the velocity field was built based on stream function and volume incompressibility condition. According to the results of finite element simulation, some parameters of dog-bone shape and rolling force are fitted. Li X et. al [17] used a continuous symmetric parabola to describe dog-bone shape and established a 2D kinematically admissible continuous velocity field as well as the corresponding upper deformation power functional. The results are verified by experiment data and traditional models. Simplified the vertical rolling process as a 2D plastic flow, Liu et. al [18-19] established sine and parabolic dog-bone models respectively. Based on rigid-plastic theory, volume invariant condition and the property of flow function, the total power functional is deduced. After minimizing the total power, the deformation shape parameters and rolling force were obtained, which are basically consistent with the measured values. On this basis, the 3D mathematical model is established by using dual-stream function method. The results shown the improvement of accuracy and application [20-22].

Through the analysis of the above literatures, it can be seen that the theoretical study on flat rolling is complete and indepth, however, lack of research on vertical rolling. The calculation results are far from each other. In addition, the semi theoretical and 2D model have a limited application. Models can not be in good agreement with reality especially in big reduction rate. Although dual-stream function model performs better, it brings a great difficulty to the selection of velocity field and the calculation of power. $\Gamma$ function and parabola function are used to describe dog-bone shape. Then, a new global weighted velocity field with the corresponding strain rate field is successfully proposed to analyze 3D deformation in vertical rolling process. Based on upper bound analysis, the rolling force and dog-bone deformation are solved numerically. The calculated results are compared with experimental and theoretical models, which verified the effectiveness and advantage of the presented weighted model. Finally, the mechanical parameters and edge deformation are studied by using the established model.

\section{Vertical rolling model}

\subsection{Analysis of plastic deformation and basic assumptions}

Vertical-horizontal rolling process in hot tandem rolling is shown in Fig. 1. The continuous casting slab passes through the vertical roller gap at a certain initial speed. The width is reduced in order to achieve the requirement of horizontal rolling. 
During vertical rolling, a 3D plastic deformation occurred, which result in obvious bulge at the edge of the steel slab. But the deformation is mainly restricted in a small zone while the middle part in width direction remains unchanged because of the high ratio of width to thickness. The rolled cross section can be regarded as a "dog-bone", as the insert of Fig.1 illustrated. The rolling velocity is $v_{0}$. The initial thickness of steel slab is given by $H_{0}$. The width of the steel slab before and after rolling are given by $W_{0}$ and $W_{1}$ respectively.

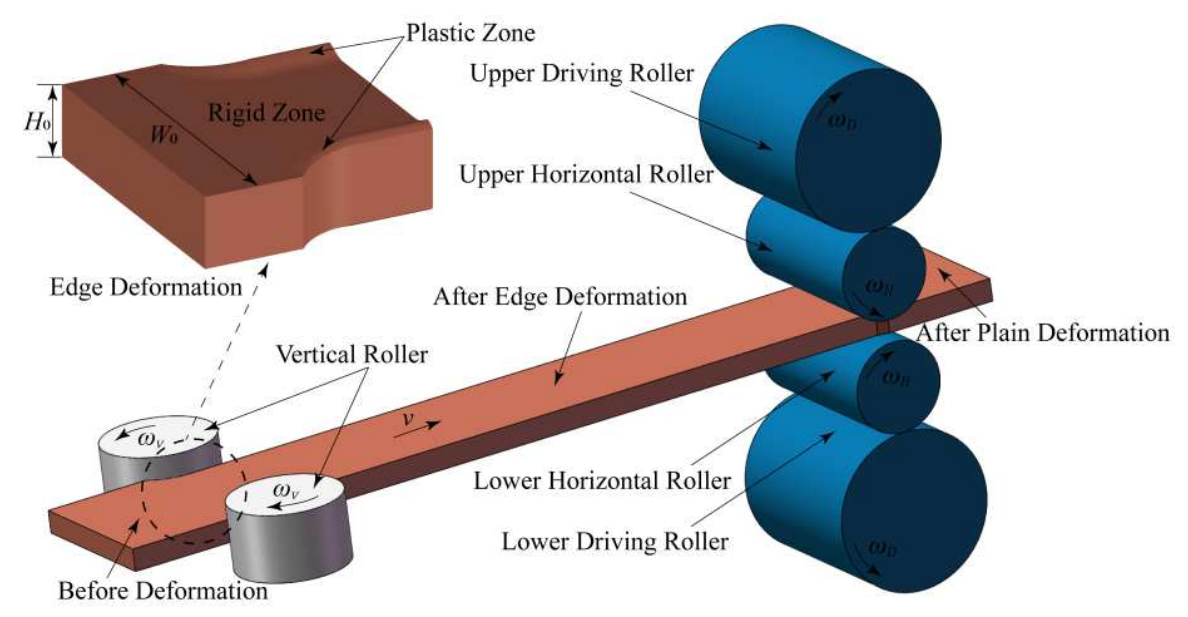

Fig.1 The schematic diagram of vertical-horizontal rolling process

Before the mechanical analysis of vertical rolling process, the following assumptions are given:

(1) The vertical rollers are rigid bodies and perpendicular to the rolling direction without assembly error. The radial displacement of rollers in rolling process is ignored. (2) The steel slab is analyzed as rigid-plastic material whose microstructure is uniform and isotropic. The upper and lower as well as the left and right parts of the slab are symmetrical with consistent force and plastic deformation rules. (3) There is no tension, and the bite zone material is in steady stage without width lose. (4) The influences of environmental factors (temperature, etc) on mechanical properties are very small and not considered.

\section{$2.2 \Gamma$-parabola function dog-bone model}

According to the symmetry of deformation, a quarter of the deformation zone is selected for mechanical analysis. As shown in Fig.2, a 3D coordinate system is established. The center of entrance cross section is chose as the coordinate origin and coordinate axis $\mathrm{x}, \mathrm{y}$ and $\mathrm{z}$ represent the rolling, thickness and width directions respectively.

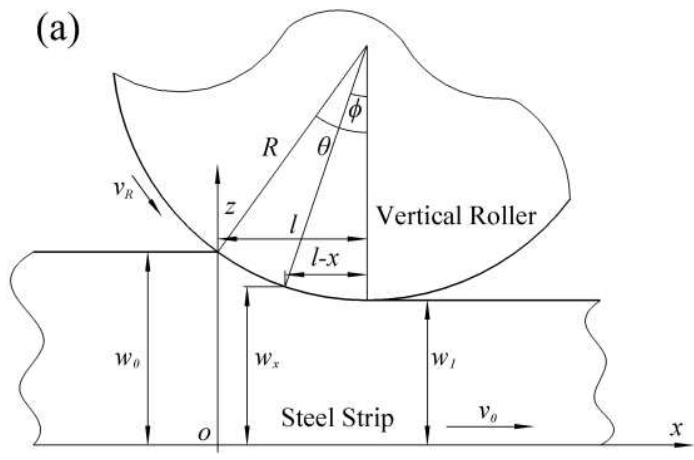

(a) The horizontal section of bite zone

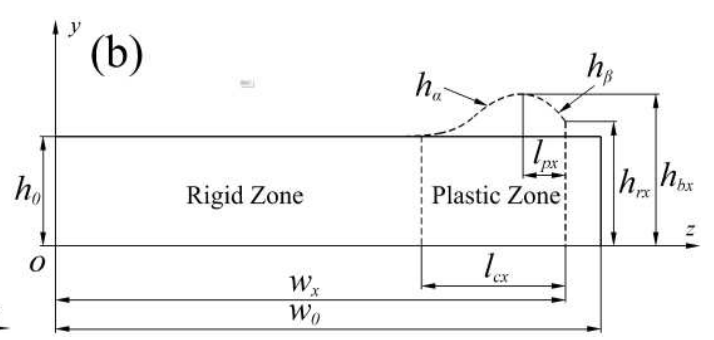

(b) The formation of dog-bone shape

Fig. 2 The section of rolling zone

In Fig. 2(a), half of the slab width before and after rolling are given by $w_{0}$ and $w_{1}$ respectively. The unilateral width reduction $\Delta w=w_{0}-w_{1}$. The vertical roller radius is denoted by $R$. The bite angle $\theta=\cos ^{-1}\left(\frac{R-\mathrm{V} w}{R}\right)$. The projected length of the roller-slab contact arc in the rolling direction $l=R \sin \theta$. Half of the width, the unilateral width reduction, and 
the contact angle at any position in bite zone are $w_{x}=w_{1}+R-\sqrt{R^{2}-(l-x)^{2}}, \Delta w_{x}=w_{0}-w_{x}$, and $\phi=\sin ^{-1}\left(\frac{l-x}{R}\right)$ respectively. The relationship between $d x$ and $d \phi$, and the first and second order derivative equation of $w_{x}$ are expressed as follow:

$$
\begin{aligned}
& d x=-R \cos \phi d \phi \\
& w_{x}^{\prime}=-\frac{l-x}{\sqrt{R^{2}-(l-x)^{2}}}=-\tan \phi \\
& w_{x}^{\prime \prime}=\frac{R^{2}}{\left[R^{2}-(l-x)^{2}\right]^{\frac{3}{2}}}
\end{aligned}
$$

As Fig. 2(b) show, the stem area of dog-bone can be regarded as rigid area while the end part is plastic area. The half thickness of steel slab in bite zone is given by $h_{(x, z)}$. In deformation zone, $h_{b x}, h_{r x}, l_{c x}$ and $l_{p x}$ represent peak height, edge height, peak position, and the length of dog-bone respectively.

Take the peak of dog-bone as the boundary to divide the dog-bone into two parts. Use $\Gamma$ function and parabola function to describe the half thickness of dog-bone in center part $\left(0<z<w_{x}-2 d_{x}\right)$ and edge part $\left(w_{x}-2 d_{x}<z<w_{x}\right)$ respectively, and assume an inverse proportion between the increase value of half thickness at dog-bone edge $\Delta h_{r}$ and the friction factor $m$. Then, two parts of the dog-bone thickness are shown as follow:

$$
\begin{aligned}
& h_{\alpha}=h_{0}+k h_{0} \frac{\Delta w_{x}}{d_{x}}\left(\frac{w_{x}-z}{d_{x}}\right)^{2} e^{-\left(\frac{w_{x}-z}{d_{x}}\right)} \\
& h_{\beta}=h_{0}+\frac{4 k}{e^{2}} h_{0} \frac{\Delta w_{x}}{d_{x}}-\frac{m k}{e^{2}} h_{0} \frac{\Delta w_{x}}{d_{x}}\left(\frac{w_{x}-z}{d_{x}}-2\right)^{2}
\end{aligned}
$$

where $k$ is the undetermined constant, $m$ is the friction factor, and $d_{x}=d+\frac{1}{6}\left(w_{x}-w_{1}\right)$ [18] expresses half distance from peak position to slab edge at arbitrary cross section.

The obtained expressions of dog-bone model satisfy the following boundary conditions:

$$
\begin{aligned}
& h_{\alpha}(0, z)=h_{\beta}(0, z)=h_{0} \\
& h_{\alpha}\left(x, w_{x}-2 d_{x}\right)=h_{\beta}\left(x, w_{x}-2 d_{x}\right) \\
& \left.\frac{\partial h_{\alpha}(x, z)}{\partial z}\right|_{z=w_{x}-2 d_{x}}=\left.\frac{\partial h_{\beta}(x, z)}{\partial z}\right|_{z=w_{x}-2 d_{x}}=0 \\
& h_{\alpha}\left(l, w_{x}-2 d_{x}\right)=h_{\beta}\left(l, w_{x}-2 d_{x}\right)=h_{0}+\frac{4 k}{e^{2}} h_{0} \frac{\Delta w_{x}}{d_{x}}=h_{b x} \\
& h_{\beta}\left(l, w_{1}\right)=h_{0}+\frac{4(1-m) k}{e^{2}} h_{0} \frac{\Delta w_{x}}{d_{x}}=h_{r x}
\end{aligned}
$$

\subsection{Velocity field and strain rate field of plastic flow}

Because of the contact of vertical roller and the resulting friction, there will be a certain change of rolling direction velocity at the edge of the slab, especially under big reduction rate. In previous studies [17-19], it seems feasible to simplify 
vertical rolling process as 2D plastic deformation, however, also bring a few impacts on accuracy. Considering 3D plastic flow, two simple deformation velocity fields are established. The kinematically admissible velocity field is determined by using global weighted method.

(1) Velocity field I

For velocity field I assumes a constant rolling direction velocity $v_{0}$, the whole reduced metal flows to thickness direction. The velocity field and strain rate field can be obtained according to the property of stream function, incompressibility condition as well as the boundary condition $\left(w_{\alpha}\left(w_{x}-2 d_{x}\right)=w_{\beta}\left(w_{x}-2 d_{x}\right)\right.$ ). The velocity field and strain rate field in center part and edge part are calculated respectively as follow:

$$
\begin{aligned}
& \left\{\begin{array}{l}
v_{\alpha x \mathrm{I}}=v_{0} \\
v_{\alpha y \mathrm{I}}=\frac{v_{0} y}{h_{0}} \frac{\partial h_{\alpha}}{\partial x}=k v_{0} y\left(t_{x}^{\prime} u^{2}-t u_{x}^{\prime} u^{2}+2 t u_{x}^{\prime} u\right) e^{-u} \\
v_{\alpha z \mathrm{I}}=v_{0} \frac{\partial w_{\alpha}}{\partial x}=k v_{0}\left[w_{x}^{\prime}\left(u^{2}+2 u+2\right)+\Delta w_{x} u_{x}^{\prime} u^{2}\right] e^{-u}
\end{array}\right.
\end{aligned}
$$

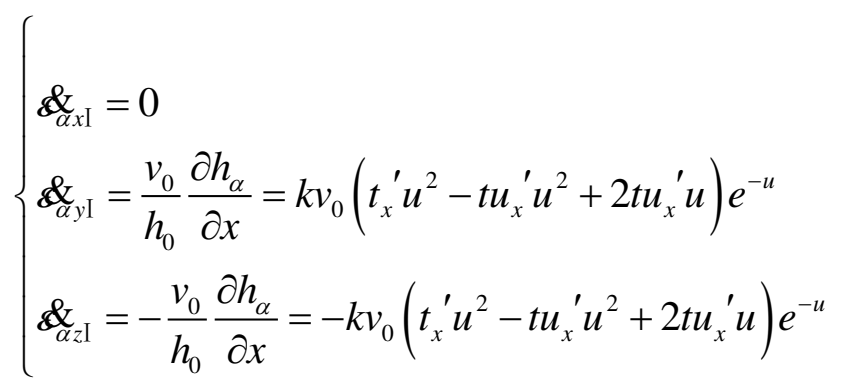

$$
\begin{aligned}
& \left\{\begin{array}{l}
v_{\beta x \mathrm{I}}=v_{0} \\
v_{\beta y \mathrm{I}}=\frac{v_{0} y}{h_{0}} \frac{\partial h}{\partial x}=\frac{k}{e^{2}} v_{0} y\left[4 t_{x}^{\prime}-m t_{x}^{\prime}(u-2)^{2}-2 m t(u-2) u_{x}^{\prime}\right] \\
v_{\beta z \mathrm{I}}=v_{0} \frac{\partial w}{\partial x}=\frac{4 k}{e^{2}} v_{0}\left(\Delta w_{x} u_{x}^{\prime}+\frac{9}{2} w_{x}^{\prime}-w_{x}^{\prime} u\right)+\frac{m k}{e^{2}} v_{0}\left[\frac{1}{3} w_{x}^{\prime}(u-2)^{3}-\Delta w_{x}(u-2)^{2} u_{x}^{\prime}\right]
\end{array}\right. \\
& \left\{\begin{array}{l}
\&_{x \mathrm{I}}=0 \\
\&_{\partial \mathrm{yI}}=\frac{v_{0}}{h_{0}} \frac{\partial h}{\partial x}=\frac{k}{e^{2}} v_{0}\left[4 t_{x}^{\prime}-m t_{x}^{\prime}(u-2)^{2}-2 m t(u-2) u_{x}^{\prime}\right] \\
\&_{z \mathrm{I}}=-\frac{v_{0}}{h_{0}} \frac{\partial h}{\partial x}=-\frac{k}{e^{2}} v_{0}\left[4 t_{x}^{\prime}-m t_{x}^{\prime}(u-2)^{2}-2 m t(u-2) u_{x}^{\prime}\right]
\end{array}\right.
\end{aligned}
$$

where $u=\frac{w_{x}-z}{d_{x}}, \quad t=\frac{\Delta w_{x}}{d_{x}}, t_{x}^{\prime}=-\frac{w_{x}^{\prime} d_{x}+\Delta w_{x} d_{x}^{\prime}}{d_{x}{ }^{2}}, \quad u_{x}^{\prime}=\frac{w_{x}^{\prime} d_{x}-\left(w_{x}-z\right) d_{x}^{\prime}}{d_{x}^{2}}$ $w_{\alpha}=-k \mathrm{~V} w_{x}\left[u^{2}+2 u+2\right] e^{-u}, \quad w_{\beta}=\frac{4 k \mathrm{~V} w_{x}}{e^{2}}\left[u-\frac{9}{2}-\frac{m}{12}(u-2)^{3}\right]$ are lateral displacement functions under the assumption of plane deformation

(2) Velocity field II

In Yun's FEM [16], the influence of vertical rollers leads to a relative change of rolling direction velocity $\left(v_{x}\right)$. The 
velocity at entry section is the minimum, then increases gradually, and reaches the initial velocity $v_{0}$ again at exit, which is similar to flat rolling. Meanwhile, the change of $v_{x}$ decreases when it is near to the width middle. Therefore, the velocity field II assumes no thickness displacement as well as a parabolic change of $v_{x}$ from center to edge at the same cross section. Then, $v_{\beta z \text { II }}$ can be deduced based on Cauchy equation, incompressibility condition, and the boundary condition. The velocity field and strain rate field is given as follow:

$$
\begin{aligned}
& \left\{\begin{array}{l}
v_{\beta x \mathrm{II}}=v_{0}+\frac{3 v_{0}}{w_{x}^{2}}\left(\frac{w_{1}}{w_{x}}-1\right) z^{2} \\
v_{\beta y \mathrm{II}}=0 \\
v_{\beta z \mathrm{II}}=3 v_{0}\left(\frac{w_{1}}{w_{x}}-1\right) \frac{w_{x}^{\prime} z^{3}}{w_{x}^{3}}+v_{0} \frac{w_{x}^{\prime}}{w_{x}^{3}} z^{3} \\
\varepsilon_{\beta x \mathrm{II}}=-9 v_{0}\left(\frac{w_{1}}{w_{x}}-1\right) \frac{w_{x}^{\prime} z^{2}}{w_{x}^{3}}-3 v_{0} \frac{w_{x}^{\prime}}{w_{x}^{3}} z^{2} \\
\varepsilon_{\beta y \mathrm{II}}=0 \\
\varepsilon_{\beta z \mathrm{II}}=9 v_{0}\left(\frac{w_{1}}{w_{x}}-1\right) \frac{w_{x}^{\prime} z^{2}}{w_{x}^{3}}+3 v_{0} \frac{w_{x}^{\prime}}{w_{x}^{3}} z^{2}
\end{array}\right.
\end{aligned}
$$

Weighting velocity field and strain rate field components of Eqs. (3)-(8) in three directions with weight coefficient $g$ simultaneously, the field components become:

$$
\begin{aligned}
& \left\{\begin{array}{l}
v_{i x}=g v_{i x \mathrm{I}}+(1-g) v_{i x \mathrm{II}} \\
v_{i y}=g v_{i y \mathrm{I}}+(1-g) v_{i y \mathrm{II}} \\
v_{i z}=g v_{i z \mathrm{I}}+(1-g) v_{i z \mathrm{II}}
\end{array}\right.
\end{aligned}
$$

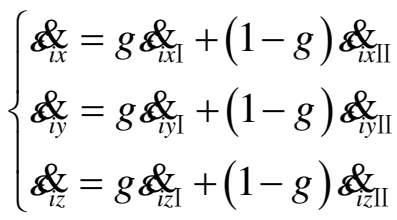

where $i=\alpha, \beta$

When $z=w_{x}$, the velocity field satisfies the boundary condition:

$$
v_{i x}=g v_{\beta x \mathrm{I}}+(1-g) v_{\beta x \mathrm{II}}=g v_{0}+(1-g)\left[v_{0}+3 v_{0}\left(\frac{w_{1}}{w_{x}}-1\right)\right]=\frac{w_{1}}{w_{0}} v_{0}
$$

The weight coefficient can be calculated as:

$g=\frac{2}{3}$

The relationship between the undetermined coefficients $k$ and $g$ is confirmed by the flow volume per second $U=$ constant at any cross section:

$$
k=\frac{e^{2}}{18-\frac{8}{3} m} g
$$

The above velocity fields satisfy the following boundary condition: 
At the entry cross section: $v_{\alpha y}(0,0, z)=v_{\beta y}(0,0, z)=0$

At the exit cross section: $v_{\alpha y}(l, y, z)=v_{\beta y}(l, y, z)=0 ; \quad v_{\alpha z}(l, y, z)=v_{\beta z}(l, y, z)=0$

At the boundary of two parts: $v_{\alpha x}\left(x, y, w_{x}-2 d_{x}\right)=v_{\beta x}\left(x, y, w_{x}-2 d_{x}\right)$

$$
\begin{aligned}
& v_{\alpha y}\left(x, y, w_{x}-2 d_{x}\right)=v_{\beta y}\left(x, y, w_{x}-2 d_{x}\right) \\
& v_{\alpha z}\left(x, y, w_{x}-2 d_{x}\right)=v_{\beta z}\left(x, y, w_{x}-2 d_{x}\right)
\end{aligned}
$$

The strain rate fields satisfy the incompressibility condition:

$\&_{\alpha x}+\underset{\alpha y}{\&}+\underset{\alpha z}{\&}=0 ; \quad \& \&_{b x}+\& \&_{b y}+\& \&_{z z}=0$

\subsection{Twin shear stress yield criterion}

Twin shear stress (TSS) yield criterion [23] locus on the $\pi$-plane is shown in Fig. 3, which is obviously the circumscribed hexagon of Mises locus. TSS yield criterion assumes that yielding begins when the Haigh Westergaard stress space are $\left(\sigma_{1} \geq \sigma_{2} \geq \sigma_{3}\right)[24]:$

$$
\begin{aligned}
& \tau_{12}+\tau_{13}=\sigma_{1}-\frac{1}{2}\left(\sigma_{2}+\sigma_{3}\right)=\sigma_{s}, \text { if } \sigma_{2} \leq \frac{1}{2}\left(\sigma_{1}+\sigma_{3}\right) \\
& \tau_{13}+\tau_{23}=\frac{1}{2}\left(\sigma_{1}+\sigma_{2}\right)-\sigma_{3}=\sigma_{s}, \text { if } \sigma_{2} \geq \frac{1}{2}\left(\sigma_{1}+\sigma_{3}\right)
\end{aligned}
$$

The corresponding specific plastic work rate is:

$$
D(\underset{i}{\&})=\frac{2}{3} \sigma_{s}(\underset{\max }{\&}-\underset{\min }{\&})
$$

where $\underset{\max }{\&}$ and $\underset{\min }{\&}\left(s^{-1}\right)$ are the maximum and minimum principal strain rates respectively.

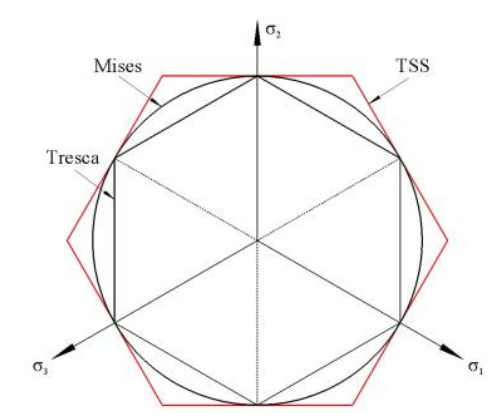

Fig. 3 TSS yield locus on the $\pi$-plane

\subsection{Total power functional model}

According to the first variational principle of rigid-plastic material, the total power functional consists of three parts:

$$
J^{*}=W_{i}^{\&}+W_{s}^{\&}+W_{f}^{\&}
$$

where $W_{i}^{\&}, W_{s}^{\&}, w_{f}^{\&}$ represent plastic deformation power, shear power and friction power respectively.

According to TSS yield criterion, the plastic deformation power is:

$$
\begin{aligned}
& W_{i}^{\&}=\iiint_{V} D(\underset{i j}{\&}) d V=\frac{2}{3} \sigma_{s} \iiint_{V} \underset{\max }{\mathbb{\&}}-\mathbb{d}_{\min } d V \\
& =\frac{2}{3} \sigma_{s} \int_{0}^{l} \int_{0}^{w_{x}-2 d_{x}} \int_{0}^{h_{\alpha}} \underset{\max }{\&}-\underset{\min }{\&} d y d z d x+\frac{2}{3} \sigma_{s} \int_{0}^{l} \int_{w_{x}-2 d_{x}}^{w_{x}} \int_{0}^{h_{\beta}} \underset{\max }{\stackrel{\&}{\&} \underset{\min }{\&}} d y d z d x
\end{aligned}
$$

The tangential velocity discontinuity exists at entry section. The shear power is:

$$
W_{s}^{\&}=\int_{S_{0}} \tau_{s}\left|\Delta v_{s}\right| d S=4 \tau_{s} \int_{0}^{w_{0}} \int_{0}^{h_{0}} \sqrt{\left(\left.v_{y}\right|_{x=0}\right)^{2}+\left(\left.v_{z}\right|_{x=0}\right)^{2}} d y d z
$$


where $\tau_{s}=\sigma_{s} / \sqrt{3}$ is the shear yield strength.

According to the boundary condition of velocity field, at exit section there is:

$\left.w_{x}^{\prime}\right|_{x=l}=0,\left.\Delta v_{y}\right|_{x=l}=\left.\Delta v_{z}\right|_{x=l}=0$

The velocity field is continuous without shear power.

On the contact surface, the friction is calculated based on constant friction theory, which can be expressed as:

$$
\tau_{f}=m \tau_{s}
$$

The velocity discontinuity and area element are:

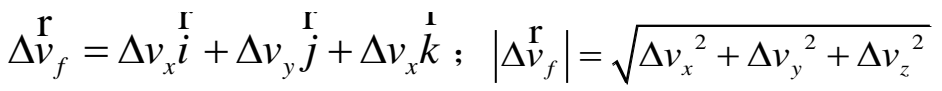

$$
\begin{aligned}
& d s=\sqrt{1+\left(\frac{d z}{d x}\right)^{2}+\left(\frac{d z}{d y}\right)^{2}} d x d y=\sqrt{1+\left(h_{x}^{\prime}\right)^{2}} d x d y=\sec \alpha d x d y
\end{aligned}
$$

Noting that friction shear stress is always collinear vector with the velocity discontinuity on the interface. The friction power can be obtained by collinear vector inner product:

$$
\begin{aligned}
W_{f}^{\&} & =\int_{S_{f}}\left|\tau_{f}^{\mathrm{r}} \| \Delta v_{f}^{\mathrm{r}}\right| \cos \left(\underset{\tau_{f}}{\mathrm{r}}, \Delta v_{f}^{\mathrm{r}}\right) d s \\
& =4 m \tau_{s} \int_{0}^{l} \int_{0}^{h_{r x}} \sqrt{\left.v_{y}\right|_{z=w_{x}}{ }^{2}+\left(\left.v_{x}\right|_{z=w_{x}}-v_{R} \cos \alpha\right)^{2}+\left(\left.v_{z}\right|_{z=w_{x}}-v_{R} \sin \alpha\right)^{2}} \sec \alpha d y d x
\end{aligned}
$$

Substituting Eqs. (13), (14) and (16) into Eq. (12), the total deformation power functional is as follow:

$$
\begin{aligned}
& J^{*}=\frac{2}{3} \sigma_{s} \int_{0}^{l} \int_{0}^{w_{x}-2 d_{x}} \int_{0}^{h_{\alpha}} \underset{\max }{\&}-\underset{\min }{\&} d y d z d x+\frac{2}{3} \sigma_{s} \int_{0}^{l} \int_{w_{x}-2 d_{x}}^{w_{x}} \int_{0}^{h_{\beta}} \underset{\max }{\&}-\underset{\min }{\&} d y d z d x \\
& +4 \tau_{s} \int_{0}^{w_{0}} \int_{0}^{h_{0}} \sqrt{\left(\left.v_{y}\right|_{x=0}\right)^{2}+\left(\left.v_{z}\right|_{x=0}\right)^{2}} d y d z \\
& +4 m \tau_{s} \int_{0}^{l} \int_{0}^{h_{r x}} \sqrt{\left.v_{y}\right|_{z=w_{x}} ^{2}+\left(\left.v_{x}\right|_{z=w_{x}}-v_{R} \cos \alpha\right)^{2}+\left(\left.v_{z}\right|_{z=w_{x}}-v_{R} \sin \alpha\right)^{2}} \sec \alpha d y d x
\end{aligned}
$$

After parameters, such as $h_{0}, \Delta w, v_{R}, R, \sigma_{s}, m$ are given, the undetermined constant $d$ can be obtained when $J^{*}$ attains the minimum value $J_{\min }^{*}$ [25]. Substituting optimal value $d$ into Eqs. (1), (2), and (17), the dog-bone shape and total power functional are received. The rolling force per unit slab thickness $F_{0}$ can be achieved by [26]:

$$
J_{\min }^{*}=2 M \frac{v_{R}}{R}=4 h_{0} l \chi F_{0} \omega
$$

where $M$ is the rolling torque, $\chi$ is the arm factor, which is selected as 0.4 [27].

\section{Numerical research}

\subsection{The calculation and discussion of edge deformation}

Shibahara [28] carried out a vertical-horizontal rolling experiment with a simulation ratio of 1:10, in which the parameters of the fourth vertical rolling process is: $w_{0}=666.5 \mathrm{~mm}, w_{1}=640 \mathrm{~mm}, h_{0}=48.5 \mathrm{~mm}, R=400 \mathrm{~mm}$, $\omega_{R}=3 \mathrm{rad} / \mathrm{s}$. The cross section of the dog-bone shape at exit calculated by weighted model $(m=0.3)$ is compared with Shibahara's result, Xiong's model [29], Okado's model [30], Yun's semi theoretical model [16], and 2D theoretical model ( $v_{x}=v_{0}$ at any position), as shown in Fig. 4. 
The value of $h_{b}$ and $h_{r}$ predicted by weighted model are only $1 \%$ and $0.4 \%$ errors compared with Xiong's results respectively, which are also less than 4\% with Okado's and Yun's results concurrently. It is worth noting that weighted model's peak position is very close to Yun's and Xiong's results, however, comparatively further from Okado's result. This is due to the vertical roller radius, which is proved to effect dog-bone deformation in previous study [31], is ignored in exponential model fitted by Okado. Due to the consideration of rolling direction flow, the weighted model predicted less degree of dogbone deformation than 2D model obviously, which is also closer to the experiment. Based on the above analysis, the weighted model has high calculation accuracy, which is more reasonable than 2D theoretical model.

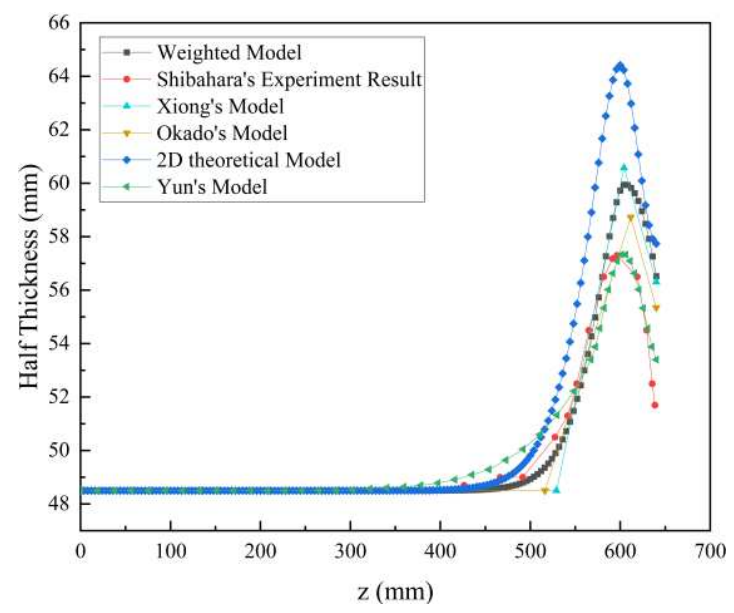

Fig. 4 Comparison of dog-bone shape acquired by the six models

When the equipment and processing parameters are determined as $m=0.3, v=1.2 \mathrm{~m} / \mathrm{s}$, the edge deformation under the width reduction of 0.03 0.05 is studied. Fig. 5-8 show the received dog-bone shape parameters compared with other models.

It can be seen from Fig. 5 that as width reduction rate increases, edge deformation moves inward with a notable and linear raise of $\frac{h_{b}}{h_{0}}, \frac{h_{r}}{h_{0}}$ and $\frac{l_{p}}{w_{0}}$. In the insert of Fig. 6(a) and Fig. 6(b), shape parameters have a similar law. Another discovery is the decrease of $\frac{h_{b}}{h_{0}}$ and $\frac{h_{r}}{h_{0}}$ in Fig. 6(a), which indicates the more uniform deformation with less plastic flow along thickness direction. Fig. 7 shows that slab width only has tiny effect on dog-bone parameters. The slight "towering" of peak can be attributed to the expand of rigid zone in middle part. Increasing the radius of vertical roller is beneficial to weaken the nonuniformity of edge deformation to a certain extent, which is verified by the calculated result as shown in Fig. 8: lower shape and the inward of the peak.
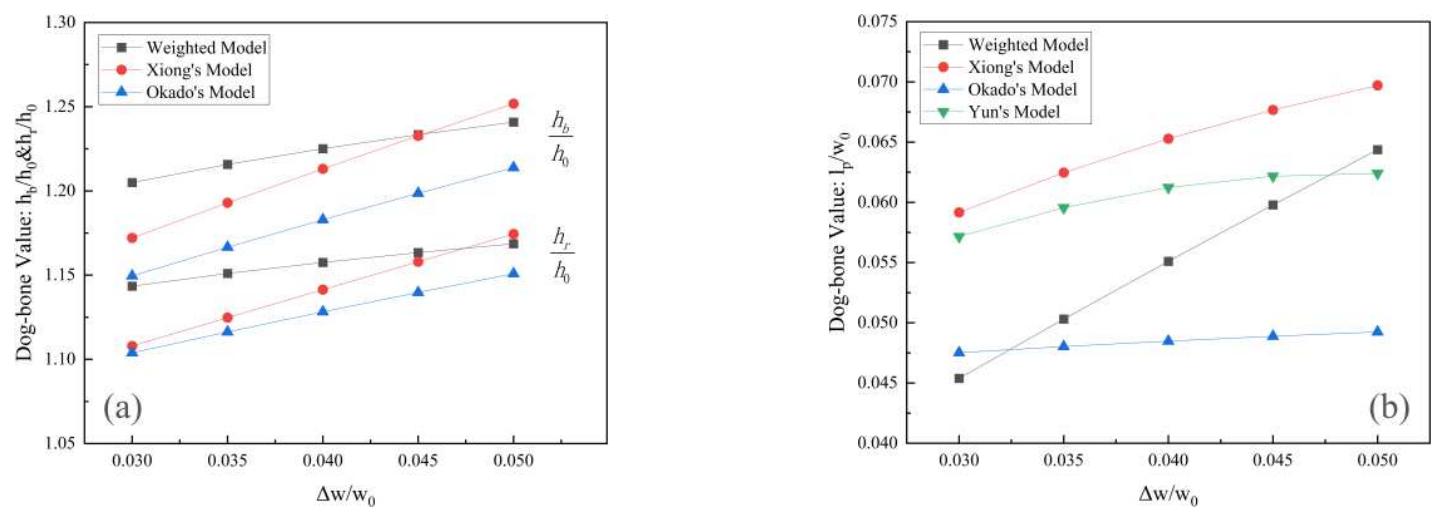

Fig. 5 The effects of $\frac{\Delta w}{w_{0}}$ on (a) height and (b) length of dog-bone $\left(\frac{h_{0}}{w_{0}}=0.1, \frac{R}{w_{0}}=0.7\right)$ 

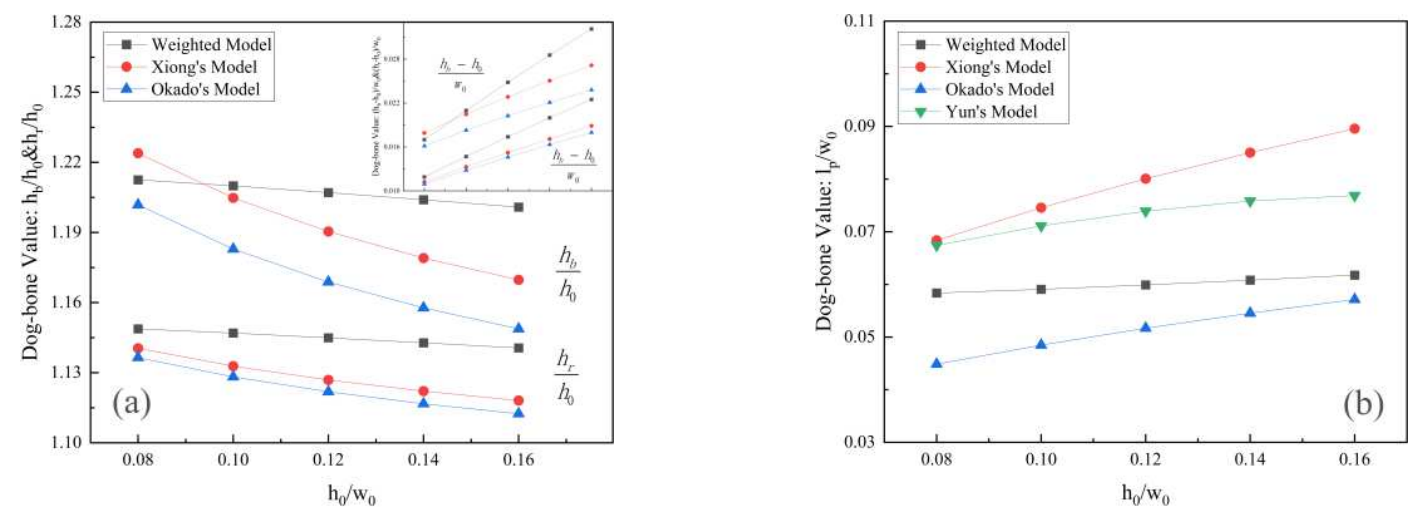

Fig. 6 The effects of $h_{0}$ on (a) heigh and (b) length of dog-bone $\left(\frac{\Delta w}{w_{0}}=0.04, \frac{R}{w_{0}}=0.9\right)$
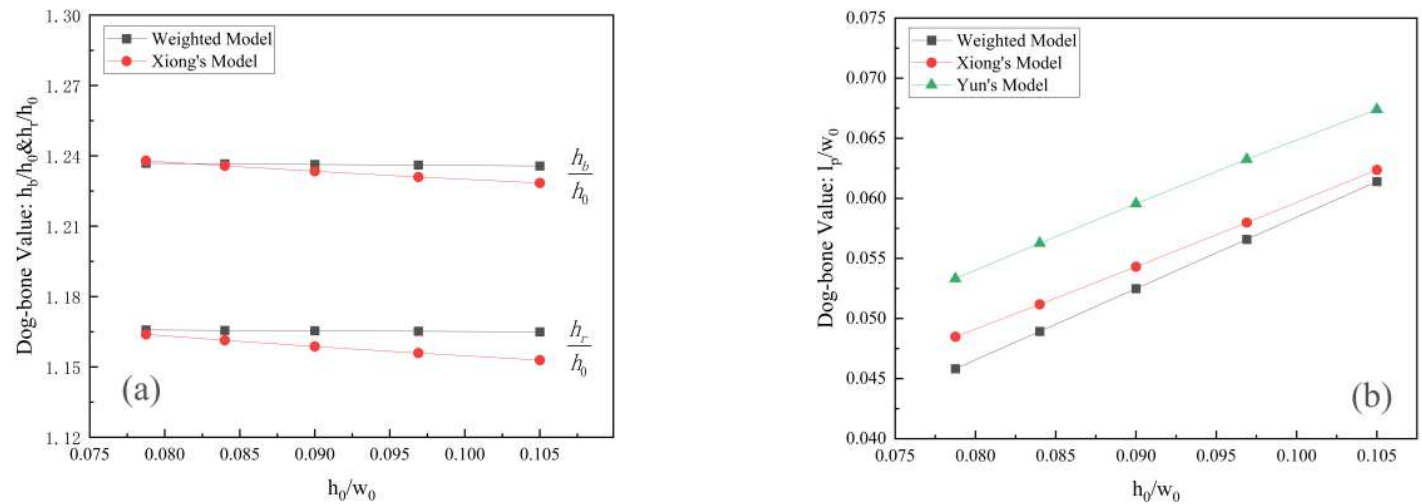

Fig. 7 The effects of $w_{0}$ on (a) heigh and (b) length of dog-bone $\left(\frac{\Delta w}{R}=0.0667, \frac{h_{0}}{R}=0.15\right)$
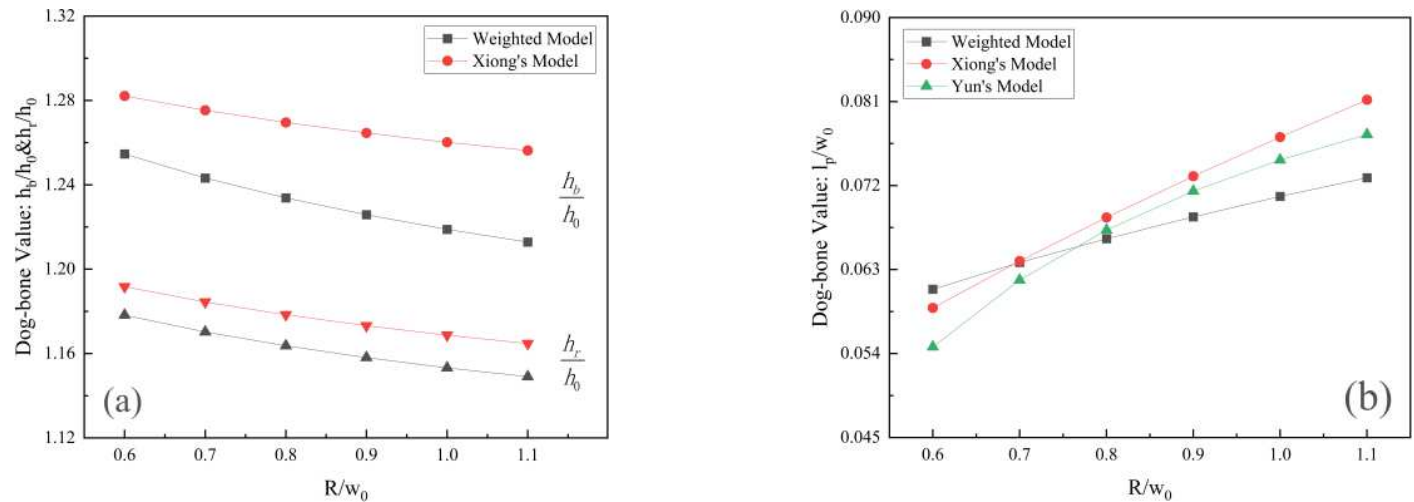

Fig. 8 The effects of $R$ on (a) heigh and (b) length of dog-bone $\left(\frac{\Delta w}{w_{0}}=0.05, \frac{h_{0}}{w_{0}}=0.08\right)$

3.2 The calculation and discussion of rolling force and power

The ratio of plastic deformation power $W_{i}^{\&}$, shear power $W_{s}^{\&}$ and friction power $W_{f}^{\&}$ in total power $J^{*}$ are compared in Fig. 9. In presented model, the velocity variation along rolling direction is considered, which lead to a larger ratio of shear power and friction power. Meanwhile, the less plastic deformation ratio can be explained by the decrease of velocity and displacement along thickness direction result from the proposal of weight coefficient. 

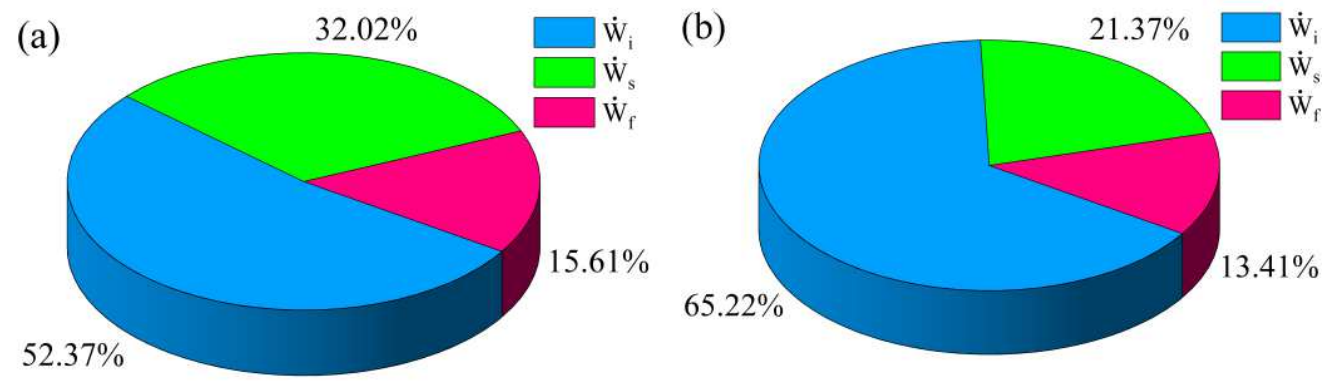

Fig. 9 The ratio of $W_{i}^{\&}, W_{s}^{\&}, W_{f}^{\&}$ in $J^{*}$ predicted by (a) weighted model and (b) dual-steam model

$$
\left(w_{0}=680 \mathrm{~mm}, \Delta w=17 \mathrm{~mm}, h_{0}=80 \mathrm{~mm}, R=580 \mathrm{~mm}\right)
$$

231 groups of experiments were carried out at the range of $w_{0}=550 \mathrm{~mm}: 800 \mathrm{~mm}, \frac{\Delta w}{w_{0}}=0.03: 0.05$, $\frac{h_{0}}{w_{0}}=0.06: 0.16, \frac{R}{w_{0}}=0.6: 1.1$. The comparison of rolling force calculated by weighted model and Yun's model is shown in Fig. 10. It can be seen that weighted model is in good agreement with Yun's model within 10\% error. Further research on reduction rate found a less error at around $4 \%$. For weighted model, the predicted dog-bone shape increases linearly with the reduction rate, which result in the uniform rise of rolling force. By contrast, Yun received a wider dog-bone zone as can be observed in Fig. 4. But the reduction rate makes weaker effect on dog-bone deformation as its increasing (shown in Fig. 5). Therefore, Yun's model shows higher rolling force while a gradually small raise.

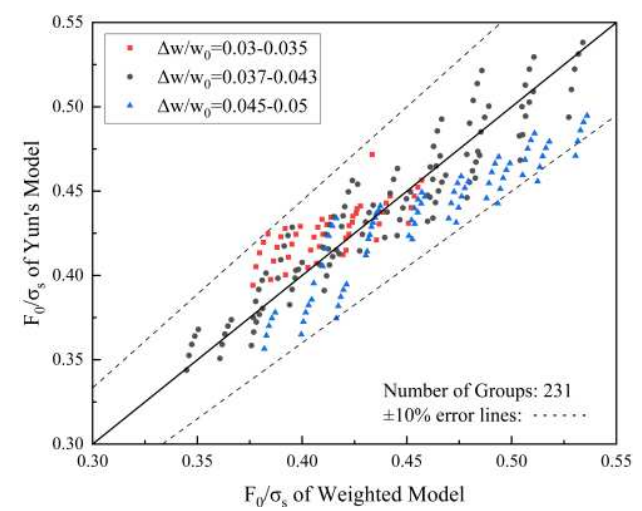

Fig. 10 Comparison of rolling force calculated by weighted model and Yun's model

When the equipment and processing parameters are determined as $m=0.3, v=1.2 \mathrm{~m} / \mathrm{s}$ and $\frac{\Delta w}{w_{0}}=0.03: 0.05$, the research on rolling force and power ratio is shown in Fig. 11 14. Both edge reduction rate and slab thickness have remarkable effects on rolling force while less impact on power ratio, as Fig. 11 12 exhibited. In Fig. 13, the wider of slab, in other words, the lower value of $\frac{h_{0}}{w_{0}}$ slightly enhance the edge deformation, which lead to the rise of rolling force and plastic deformation ratio. Fig. 14 indicates that the increase of roller radius diminishes the velocity discontinuity at entry section, which result in the limitation of shear power. Another influence is a rise in rolling force and plastic power ratio due to the expansion of deformation zone. 


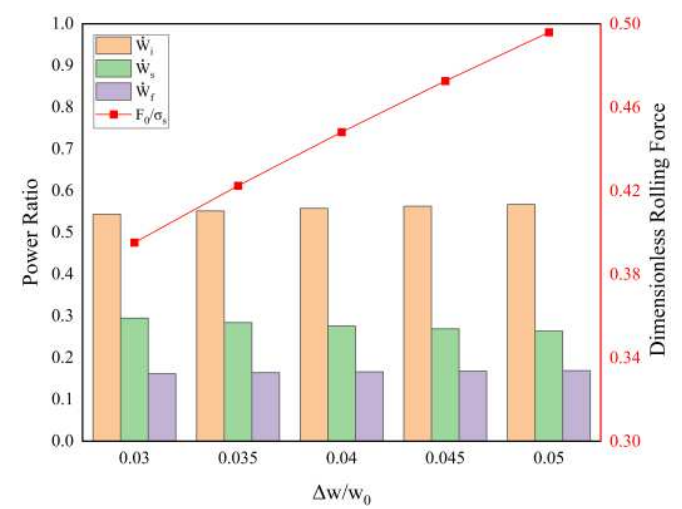

Fig. 11 The effects of $\frac{\Delta w}{w_{0}}$ on rolling force and power ratio $\left(\frac{h_{0}}{w_{0}}=0.12, \frac{R}{w_{0}}=0.9\right)$

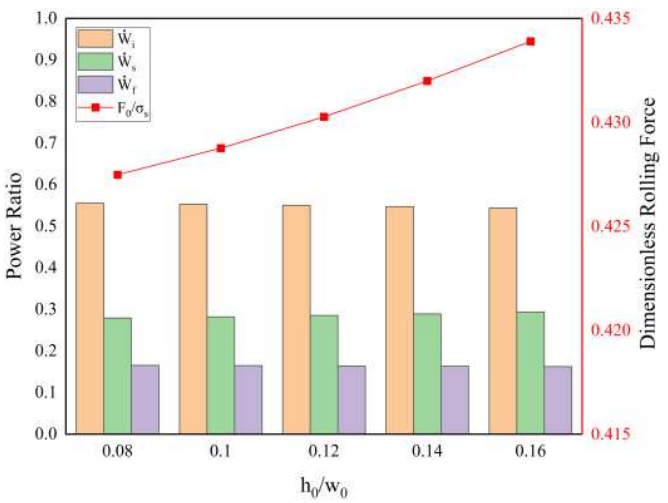

Fig. 12 The effects of $h_{0}$ on rolling force and power ratio $\left(\frac{\Delta w}{w_{0}}=0.04, \frac{R}{w_{0}}=0.8\right)$

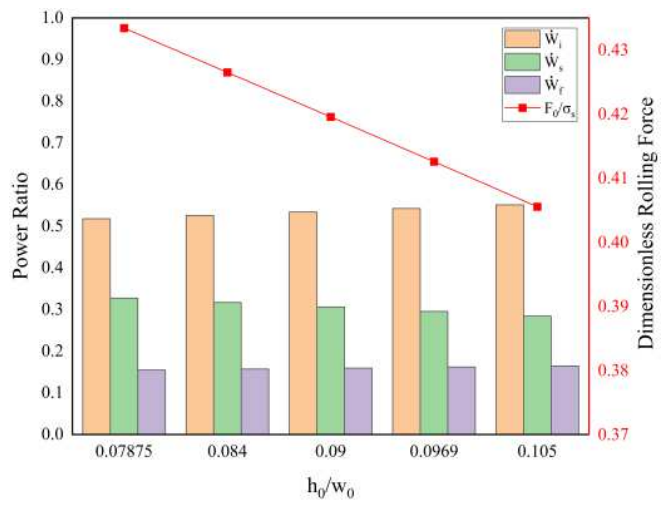

Fig. 13 The effects of $w_{0}$ on rolling force and power ratio $\left(\frac{\Delta w}{R}=0.0667, \frac{h_{0}}{R}=0.15\right)$

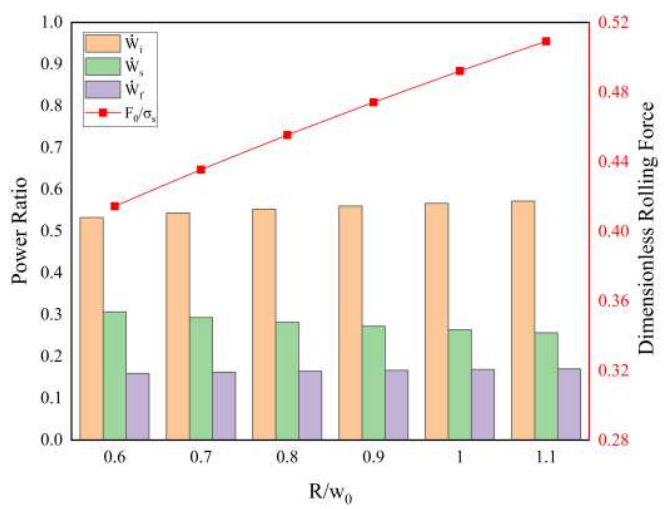

Fig. 14 The effects of $R$ on rolling force and power ratio $\left(\frac{\Delta w}{w_{0}}=0.045, \frac{h_{0}}{w_{0}}=0.14\right)$ 


\section{Conclusions}

(1) Considering the plastic flow in rolling direction, a global weighted velocity field with the corresponding strain rate field is firstly proposed to analyze edge deformation in vertical rolling based on incompressibility condition and rigid-plastic assumption. On this basis, the 3D energy model is solved by minimizing the total power functional. Then, the dog-bone parameters and rolling force are obtained.

(2) The dog-bone parameters and rolling force obtained from weighted model are compared with several theoretical and experimental models with the width reduction rate of $0.03 \sim 0.05$. The results show that the proposed model is of high prediction precision. Moreover, the influences of rolling parameters on dog-bone shape, rolling power and rolling force are studied. Width reduction rate has a noteworthy relationship with dog-bone shape and rolling force but less effect on power ratio, which is similar to initial slab thickness. The increase of slab width will aggravate the nonuniformity of edge deformation, which lead to a minor rise of rolling force and plastic power ratio. The vertical roller with bigger radius can help to control the peak height, while lead to the extension of deformation zone as well as the increase of rolling force and plastic power ratio.

(3) By specific calculation examples, it is found that the proposed weighted model accurately predicted the edge deformation and rolling force in vertical rolling process, which can satisfy the requirement in industrial production. The research can be helpful for the control of slab shape and the improvement of rolling quality. 


\section{Statements and Declarations}

The authors declare that no funds, grants, or other support were received during the preparation of this manuscript. The authors have no relevant financial or non-financial interests to disclose. 


\section{References}

1. Oh SI, Kobayashi S (1975) An approximate method for a three-dimensional analysis of rolling. Int J Mech Sci 17(4):293305. https://doi.org/10.1016/0020-7403(75)90010-7

2. Kato K, Murota T, Kumagai T (1980) Flat-Rolling of Rigid-Perfectly Plastic Solid Bar by the Energy Method. J Jpn Soc Techol Plast 21:359-369.

3. Avitzur B, Pachla W (1986) The Upper Bound Approach to Plane Strain Problems Using Linear and Rotational Velocity Fields_Part I: Basic Concepts. J Manuf Sci E-T ASME 108(4):295-306. https://doi.org/10.1115/1.3187080

4. Avitzur B, Pachla W (1986) The Upper Bound Approach to Plane Strain Problems Using Linear and Rotational Velocity Fields_-Part II: Applications. J Manuf Sci E-T ASME 108(4):307-316. https://doi.org/10.1115/1.3187080

5. Avitzur B, Gordon W, Talbert S (1987) Analysis of Strip Rolling by the Upper Bound Approach. J Manuf Sci E-T ASME 109(4):338-346. https://doi.org/10.1115/1.3187137

6. Dong YG, Zhang WZ, Song JF (2010) Theoretical and Experimental Research on Rolling Force for Rail Hot Rolling by Universal Mill. ISIJ Int 17(1):27-32. https://doi.org/10.1016/S1006-706X(10)60040-4

7. Zhang JL, Cui ZS (2011) Prediction of Velocity and Deformation Fields During Multipass Plate Hot Rolling by Novel Mixed Analytical-Numerical Method. ISIJ Int 18(7):20-27. https://doi.org/10.1016/S1006-706X(11)60085-X

8. Sun J, Liu YM, Hu YK, Wang QL, Zhang DH, Zhao DW (2016) Application of hyperbolic sine velocity field for the analysis of tandem cold rolling. Int J Mech Sci 108-109:166-173. https://doi.org/10.1016/j.ijmecsci.2016.02.004

9. Zhang DH, Cao JZ, Xu JJ, Peng W, Zhao DW (2014) Simplified Weighted Velocity Field for Prediction of Hot Strip Rolling Force by Taking into Account Flattening of Rolls. ISIJ Int 21(7):637-643. https://doi.org/10.1016/S1006706X(14)60099-6

10. Cao JZ, Zhao DW, Zhang SH, Peng W, Chen SZ, Zhang DH (2014) Analysis of Hot Tandem Rolling Force with Logarithmic Velocity Field and EA Yield Criterion. ISIJ Int 21(3):295-299. https://doi.org/10.1016/S1006706X(14)60045-5

11. Zhang SH, Song BN, Wang XN, Zhao DW (2014) Analysis of plate rolling by MY criterion and global weighted velocity field. Appl Math Model 38(14):3485-3494. https://doi.org/10.1016/j.apm.2013.11.061

12. Ma GS, Liu YM, Peng W, Yin FC, Ding JG, Zhao DW, Di HS, Zhang DH (2017) A new model for thermo-mechanical coupled analysis of hot rolling. J Braz Soc Mech Sci Eng 39(2):523-530. https://doi.org/10.1007/s40430-015-0390-9

13. Peng W, Ding JG, Zhang DH, Zhao DW (2017) A novel approach for the rolling force calculation of cold rolled sheet. J Braz Soc Mech Sci Eng 39(10):1-11. https://doi.org/10.1007/s40430-017-0774-0

14. Peng W, Zhang DH, Zhao DW (2017) Application of parabolic velocity field for the deformation analysis in hot tandem rolling. Int J Adv Manuf Technol 91:2233-2243. https://doi.org/10.1007/s00170-016-9936-y

15. Li S, Wang ZG, Guo YF (2019) A novel analytical model for prediction of rolling force in hot strip rolling based on tangent velocity field and MY criterion. J Manuf Process 47(Nov.):202-210. https://doi.org/10.1016/j.jmapro.2019.09.037

16. Yun D, Lee D, Kim J, Hwang S (2012) A new model for the prediction of the dog bone shape in steel mills. ISIJ Int 52(6):1109-1117. https://doi.org/10.2355/isijinternational.52.1109

17. Li X, Wang HY, Liu YM, Zhang DH, Zhao DW (2016) Analysis of edge rolling based on continuous symmetric parabola curves. J Braz Soc Mech Sci Eng 39(4):1-10. https://doi.org/10.1007/s40430-016-0587-6

18. Liu YM, Ma GS, Zhang DH, Zhao DW (2015) Upper bound analysis of rolling force and dog-bone shape via sine function model in vertical rolling. J Mater Process Tech 223:91-97. http://doi.org/10.1016/j.jmatprotec.2015.03.051

19. Liu YM, Zhang DH, Zhao DW, Sun J (2016) Analysis of vertical rolling using double parabolic model and stream function velocity field. Int J Adv Manuf Technol 82(5-8):1153-1161. https://doi.org/10.1007/s00170-015-7393-7 
20. Liu YM, Sun J, Zhang DH, Zhao DW (2018). Three-dimensional analysis of edge rolling based on dual-stream function velocity field theory. J Manuf Process 34(AUG.):349-355. https://doi.org/10.1016/j.jmapro.2018.06.012

21. Liu YM, Wang T, Wang ZH, Hao PJ, He DP (2020) Research on mechanics parameters of edge rolling based on dualstream function. Journal of Plasticity Engineering 27(08):172-178. https://doi.org/10.3969/j.issn.10072012.2020.08.023

22. Liu YM, Hao PJ, Wang T, Ren ZK, Zhang SH (2020) Mathematical model for vertical rolling deformation based on energy method. Int J Adv Manuf Technol 107:875-883. https://doi.org/10.1007/s00170-020-05094-3

23. Yu MH (1983) Twin shear stress yield criterion. Int J Mech Sci 25(1):71-74. https://doi.org/10.1016/00207403(83)90088-7

24. Huang WB, Zeng GP (1989) Application of twin shear stress yield criterion to solve some problems in plastic mechanics. ACTA Mech Sinica-PRC 21(2):249-256. https://doi.org/10.6052/0459-1879-1989-2-1989-302

25. Zhang SH (2016) Mechanical Principle of Plastic Forming. Metallurgical Industry Press, Beijing

26. Zhang SH, Zhao DW, Gao CR (2012) The calculation of roll torque and roll separating force for broadside rolling by stream function method. Int J Mech Sci 57(1):74-78. https://doi.org/10.1016/j.ijmecsci.2012.02.006

27. Zhao ZY (1980) Metal plastic deformation and rolling theory. Metallurgical Industry Press, Beijing

28. Shibahara T, Misaka Y, Kono T, Koriki M, Takemoto H (1981) Edger set-up model at roughing train in a hot strip mill. Tetsu-to-Hagane 67(15):2509-2151

29. Xiong SW, Zhu XL, Liu XH, Wang G, Zhang Q, Li H, Meng X, Han L (1997) Mathematical model of width reduction process of roughing trains of hot strip mills. Shanghai Metal 19(1):39-43

30. Okado M, Ariizumi T, Noma Y, Yabuuchi K, Yamazaki Y (1981) Width behaviour of the head and tail of slabs in edge rolling in hot strip mills. Tetsu-to-Hagane 67(15):2516-2525

31. Xiong SW, Liu XH, Wang GD, Zhang W (1997) Simulation of vertical-horizontal rolling process during width reduction by full three-dimensional rigid-plastic finite element method. $J$ Mater Eng Perform 6(6):757-765. https://doi.org/10.1007/s11665-997-0078-0 